\title{
OCCURRENCE OF NATURAL INFECTION WITH MYCOPLASMA HYOPNEUMONIAE AND PORCINE REPRODUCTIVE RESPIRATORY SYNDROME VIRUS (PRRSV) IN PIGS UNDER GERMAN FIELD CONDITIONS
}

\author{
M. Genzow \\ Boehringer Ingelheim Animal Health GmbH, D-55216 Ingelheim/Rhein; V.F. Ohlinger, bioScreen European \\ Veterinary Disease Management Center GmbH, Mendelstr. 11, D-48149 Münster
}

\section{Objective}

The study aimed at investigating the occurrence of antibodies against the porcine pathogens Mycoplasma hyopneumoniae (M. hyo) and PRRS virus (PRRSV) in the serum of pigs aged 10 to 25 weeks and kept under field conditions in Germany.

\section{Methodology}

As part of a broad clinical trial comparing the efficacy of two M. hyo vaccines, serological investigations took place in 40 animals of the negative control group (subdivided into 2 subgroups of 20 animals each). As the case with the remainder of the control group, these animals had previously received a single administration of $2 \mathrm{ml}$ isotonic saline deep intramuscularly in the neck at approximately 3 weeks of age. Blood samples were taken at the piglets' entry into the fattening unit (week 10 of age), in the middle of the fattening period (week 17 of age) and at the end of the fattening period (week 25 of age). Employing ELISA technology, the samples were investigated for both M. hyo and PRRSV antibodies. The pigs were not vaccinated against PRRSV.

\section{Results}

The serological investigations revealed that at the first 2 sampling time points (10 and 17 weeks of age, respectively) no antibodies against $\mathrm{M}$. hyo were present in the serum of the piglets. Only at 25 weeks of age M. hyo antibodies could be detected in $70 \%$ of the animals in subgroup 1 and in $75 \%$ of the animals in subgroup 2.

On the contrary antibodies against PRRS virus were detected in the majority of the animals at all three sampling time points. Thus at 10 weeks of age $100 \%$ of the piglets tested positive for PRRS virus antibodies. Seven weeks later, the serum of all 20 animals in subgroup 1 and of $95 \%$ of the piglets in subgroup 2 contained antibodies against PRRS virus. The situation was similar at 25 weeks of age with $85 \%$ of pigs in subgroup 1 having antibodies against PRRS virus and $100 \%$ of animals in subgroup 2.

\section{Conclusions}

The serological results obtained in the negative control animals provide evidence that the herd was infected with Mycoplasma hyopneumoniae and that this infection occurred rather late in the fattening period, i.e. at around week 20 of age. Infection with PRRS virus was demonstrated already at the first investigation time point at 10 weeks of age. This is a common finding in swine production, which is known to favour subsequent infection with other porcine pathogens. 